\title{
Cholesteatoma and Its Management
}

\author{
Ashutosh Ganesh Pusalkar ${ }^{1,2}$
}

Received: 20 August 2015/Accepted: 21 August 2015/Published online: 12 September 2015

(C) Association of Otolaryngologists of India 2015

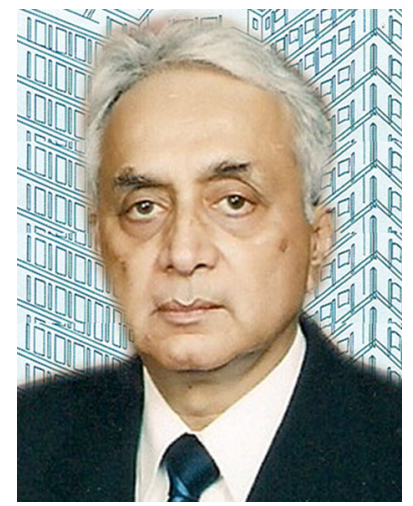

\begin{abstract}
Cholesteatoma is probably one of the very few conditions in the body with as many controversies regarding its name, definition, genesis and pathology. It is essentially a benign tumour, which pushes away the middle ear cleft mucosa and any tissues that get in its way. The indisputable starting point is the presence of an inflammatory process of the upper airways and the middle ear cleft mucosa. The growth pattern of the cholesteatoma is dictated by the site of origin and the most common sites are the pars flaccida and the postero-superior quadrant of the pars tensa. The diagnosis of cholesteatoma is essentially clinical and treatment is surgical wherein the primary aim of surgery is to provide a disease free dry ear. However, keeping with the changing clinical profile of patients
\end{abstract}

Ashutosh Ganesh Pusalkar apusalkarcl@gmail.com

1 Department of ENT, Lilavati Hospital and Research Centre, Mumbai, India

2 Department of ENT, Dr D. Y. Patil Hospital and Research Centre, Navi Mumbai, India presenting with cholesteatoma, it is important to also aim at a functionally better ear.

Keywords Cholesteatoma - Mastoidectomy · Reconstruction

\section{Etiopathogenesis}

It is important to discuss the etiology of cholesteatoma because it has relevance to its management. Many theories were put forth to explain the pathology of cholesteatoma. The most commonly held hypothesis were Eustachian tube malfunction; invasion of epithelium or migration of epithelium and metaplasia. However from a clinical point of view, these theories do not fully explain the morphological and pathological processes involved in formation of cholesteatoma and retraction pockets.

The indisputable starting point is the presence of an inflammatory process of the upper airways and the middle ear cleft mucosa. This inflammatory process when involving the posterosuperior compartment of middle ear causes problems regarding exchange of gases through the mucosa creating a negative pressure in the middle ear leading to development of retraction pocket. The inflammatory process leads to atrophy of the lamina propria of the tympanic membrane making it more susceptible to pressure changes in the middle ear. The inflammatory stimulus also leads to dysfunction of the stratified squamous epithelium of the tympanic membrane leading to accumulation of keratin into pockets. In addition local contributing factors such as local irritants triggers the squamous stratified epithelium of the external auditory canal. All these factors predispose to the genesis of a retraction pocket which could eventually lead to cholesteatoma formation [1]. 


\section{Growth Pattern of Cholesteatoma}

The growth pattern of the cholesteatoma is dictated by the site of origin. The most common sites are the pars flaccida and the postero-superior quadrant of the pars tensa, which correspond to the weaker areas of the tympanic membrane lamina propria. The pathways for enlarging cholesteatomas are specific and depend on the presence of obstacles located in the middle ear cleft.

Typically, a cholesteatoma starting from the pars flaccida will invade posteriorly, inferiorly, or anteriorly, producing a variety of complications. When it expands posteriorly, its starts from the Prussak's space and then grows towards the superior incudal space, above and lateral to the malleus head and body of the incus and then towards the aditus ad antrum to enter the mastoid air cells system. The pars flaccida cholesteatoma may grow inferiorly passing medial to the long process of the incus towards the petrous portion and the mastoid air cells system. It may grow anteriorly towards the anterior compartment of the epitympanum [1].

A cholesteatoma of the postero-superior quadrant of the pars tensa directly enters the tympanic cavity in the direction of the hypotympanum or sinus tympani, may grow forward towards the protympanum, or invade the epitympanum and mastoid.

\section{Cholesteatoma and Adjacent Bone}

The ability of cholesteatoma to attack and resorb adjacent bone is clear and this constitutes one of the characteristics of its pathological process. Numerous mechanisms have been implicated in this bone resorption, such as pressure necrosis, chronic osteomyelitis, osteoclastic resorption and biochemical enzymetic resorption. These may act alone or, more probably, in combination. It has been demonstrated that bone erosion only occurs as a result of the action of multinucleate osteoclasts, in areas where the cholesteatoma is in contact with the bone. This acts as a local stimulus for osteoclast activation. This explains why facial nerve paraesis is not clinically elicited in all cases of fallopian canal dehiscence. The facial nerve is affected due to inflammation of facial sheath rather than canal erosion and actual destruction of the facial nerve is almost never seen.

\section{Clinical Profile and Management}

The clinical appearance of middle ear cholesteatoma is changing in our country rapidly. Acute necrotic otitis media due to measles or tuberculosis, diphtheria and other viral infections are on the decline these days. Improvement in health care, improvement in good immunization, good prohylaxis and prompt antibiotic therapy of acute excenthmas in childhood perhaps is the result of this recent change. Though the incidence of secondary cholesleatoma with posterior superior marginal or subtotal defect of tympanic membrane is high, the so called primary epitympanic cholesteatoma of pars flaccida are being seen in considerably large number. As there are no symptoms in the beginning of sharpnel cholesteatoma the disorder is often detected at a later age and these patients complain of ear trouble for the first time in their 3rd or 4th decade of life. The examination reveals cholesteatoma originating in pars flaccida. A thorough examination of the tympanic membrane under microscope is important so that this pathology may not be missed.

The diagnosis of cholesteatoma is an essentially clinical with microscope examination and hearing assessment playing a key role probably aided today by CT scans. However in cases of congenital cholesteatoma, an HRCT temporal bone or preferably an MRI temporal bone is necessary for diagnosis.

The surgical techniques followed for cholesteatoma surgery are:

(1) Antero posterior approach (Fig. 1)

(2) Posterior tympanotomy (Fig. 2)

(3) Combined approach

(4) Reconstruction posterior wall

Cartilage

Replacing posterior wall (Fig. 3)

Aloplastic material

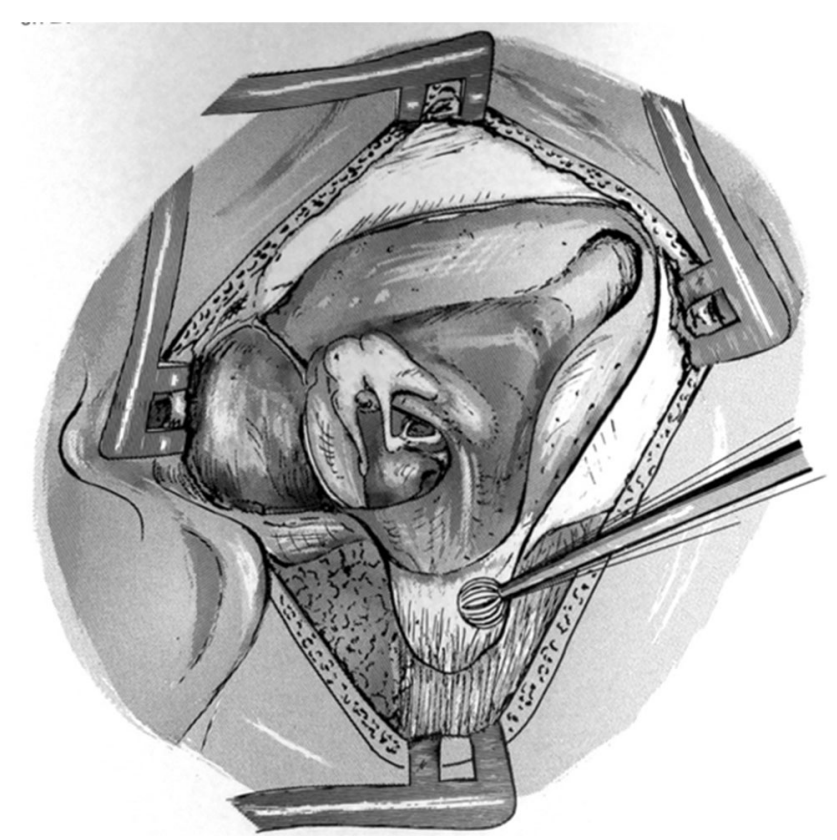

Fig. 1 Modified radical mastoidectomy- Anterior posterior approach (Figure courtesy of Hildmann and Sudhoff) 


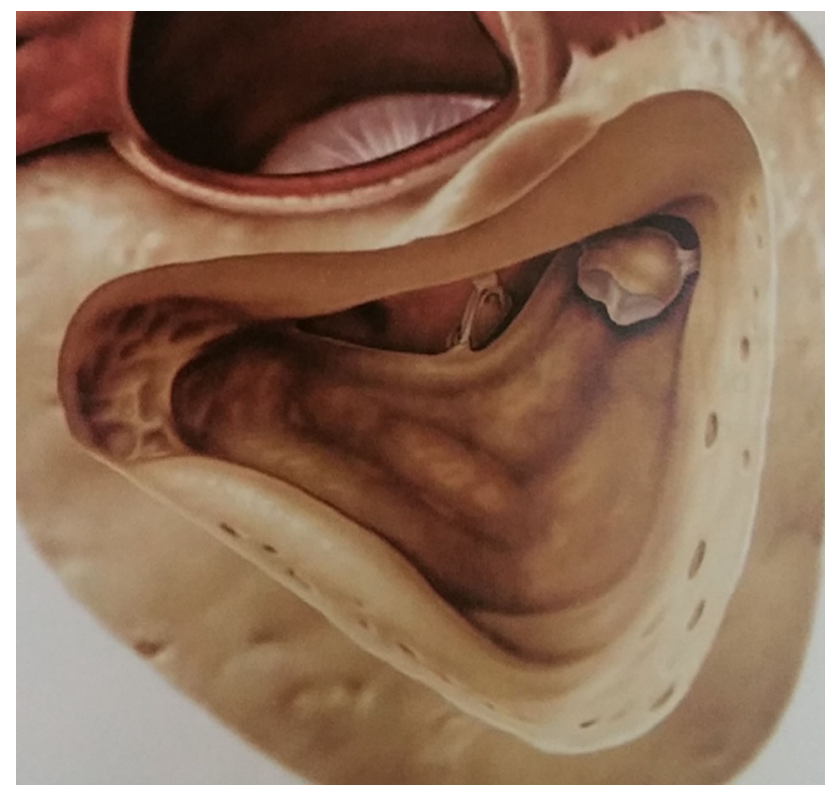

Fig. 2 Posterior tympanotomy (Figure courtesy of Hildmann and Sudhoff)

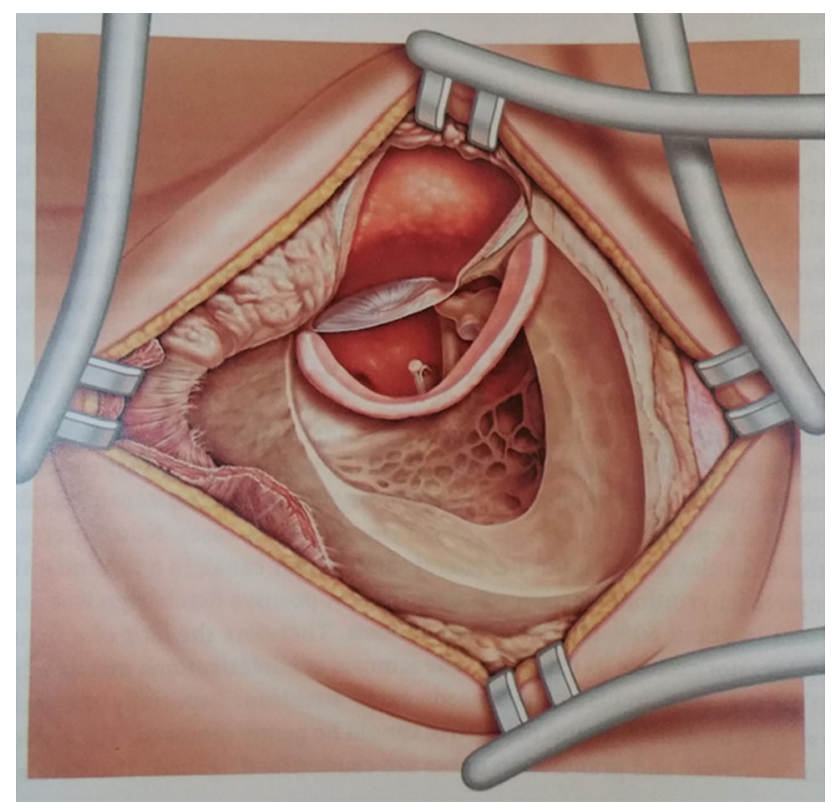

Fig. 3 Reconstruction posterior meatal wall (Figure courtesy of Hildmann and Sudhoff)

A small attic cholesteatoma in a 40 year old patient with a poorly pneumatised or cellular mastoid calls for one surgical technique and the cholesteatoma of a child with well pneumatised mastoid needs quite another form of surgical intervention. Some cases lay between these two extremes, hence the choice of surgical procedure becomes difficult. It is always necessary to keep in mind the socioeconomic condition and the educative background of the parents of the child before deciding on surgical approach.
There are no two opinions that a wide opening of the mastoid to the external auditory meatus, the attico antrostomy is still today a most reliable method of surgical treatment of cholesteatoma. It has a big advantage of safety. The disadvantage could be for a person who is a competitive swimmer or a diver. A combined anterior and posterior approach may be adopted to achieve not only removal of the pathological process but also to retain a large middle ear volume and the physiological self cleaning capacity of the ear.

The drawbacks of the so called 'closed techniques' is the recurrence of cholesteatoma in attic or in the retrotympanic space due to incomplete removal of the squamous epithelium. The classical closed technique as described by Professor Jensen has recurrence rate of as high as $35 \%$. In cases of cholesteatoma the lateral attic wall is already destroyed and after completion of posterior tympanotomy this defect continues to persist. In addition to high recurrence rate another danger is the development of epitympanic retraction pocket due to original lateral attic wall defect which in course of months can give rise to new cholesteatoma. 'Cody' in 1971 called this retraction pocket as precholesteatoma. It is interesting to note that such retraction pocket are rarely seen after stapes or facial nerve surgery inspite of substantial bony defects in lateral attic wall.

Almost all cholesteatomas are managed surgically. The conservative management is followed for a specific short duration in certain cases. In cases where a patient presents with acute mastoiditis with postaural abscess, the abscess should be incised and drained. A mastoid surgery should not be attempted during this acute stage because in most of the cases patients are toxic. After incision and drainage of abscess the patient should be kept on antibiotics for 2 weeks, general condition improved and then operated for classical radical mastoidectomy. In chronic mastoditis with post aural fistula the surgical procedure carried out is the same, however broad spectrum antibiotics should be prescribed for a long time.

Secondary acquired cholesteatoma is the largest group of patients and the diagnosis is always clinical. The two main concerns of management are permanent irradication of the disease and improvement of hearing. As far as irradication of the disease is concerned, in all cases attico antrostomy approach can be followed leaving behind small cavity exteriorised to the external auditory meatus.

The bony defects caused either by surgeon or cholesteatoma should always be reconstructed. Reconstruction of lateral attic wall in an epitympanic cholesteatoma should be done only if the retraction pocket can be elevated completely without breakdown of the squamous epithelium. If this cannot be done, it is best to opt for a modified radical approach and expose the cavity. 
The reconstruction of sound conducting system depends on the problems encountered. All these problems could be isolated or in combination. Actually there is no difference in principal of reconstruction of ossicular chain in patients with or without cholesteatoma. Ossicular reconstruction can be either primary at the time of the first surgery or staged to be done at a later time once the ear is disease free. The cases where staged reconstruction is preferred are congenital cholesteatomas, cases with middle ear or mucosal involvement, or in cases operated via a posterior tympanotomy approach.

When the long process of incus is destroyed, the incus is removed along with head of the malleus because if the head is left behind the region anterior to the head always becomes a site of recurrence. The reconstruction is made using titanium partial ossicular replacement prosthesis. When the suprastructure of the stapes is destroyed, squamous epithelium is very carefully removed followed by reconstruction using titanium total ossicular replacement prosthesis. The cartilage 'shoe' technique can be employed for stabilising the total ossicular replacement prosthesis in the oval window niche.

If the handle of malleus is fore-shortened, in such circumstances the tensor tympani tendon is cut to aid insertion of the ossicle. However, in most cases it is advisable to remove the entire malleus to facilitate a simpler ossicular reconstruction. Also the sound transmission following such a reconstruction is better. On some occasions the head of the stapes is barely at the facial ridge. Introduction of ossicle makes it too high. In this case tragal cartilage with perichondrium is kept between the stapes and the handle of malleus, which helps in both ways, as a sound transmitting structure as well as closure of the perforation. Lastly in case of obliterated eustachian tube with full epithialisation no attempt is made to improve the hearing.

Recurrence following cholesteatoma surgery has been reported. In addition there are certain number of patients where cavites go on discharging inspite of being small, causing nuisance to the patient as well as the surgeon. Mostly these are the patients in whom the cholesteatoma grows in a finger like process and the cavities require repeated cleaning.

\section{Conclusions}

Cholesteatoma in the middle ear cleft is a dynamic process which undergoes progressive growth and may cause subsequent serious local problems. The pathological feature of cholesteatoma is that it erodes the bone of the middle ear cleft by the induction of osteoclasts. Although the preference for the best approach for cholesteatoma surgery is still under debate, the primary aim of surgery should be to provide a disease free dry ear. However, keeping with the changing clinical profile of patients presenting with cholesteatoma, it is important to start managing cholesteatoma not only from the point of view of safe and dry ear but also in addition a functionally better ear.

\section{References}

1. Bernard MPJ (1999) Pathogenesis of acquired cholesteatoma. In: Ars B (ed) Pathogenesis of cholesteatoma. Kugler Publications, Amsterdam, pp 6-15 